\title{
Cyclin D1 expression in prostate carcinoma
}

\author{
R.A. Pereira ${ }^{1}$, R.C. Ravinal ${ }^{1}$, R.S. Costa ${ }^{1}$, M.S. Lima ${ }^{1}$, S. Tucci ${ }^{2}$, V.F. Muglia ${ }^{3}$, \\ R.B. Dos Reis ${ }^{2}$ and G.E.B. Silva ${ }^{1}$ \\ ${ }^{1}$ Departamento de Patologia, Faculdade de Medicina de Ribeirão Preto, Universidade de São Paulo, Ribeirão Preto, SP, Brasil \\ ${ }^{2}$ Divisão de Urologia, Departamento de Cirurgia e Anatomia, Faculdade de Medicina de Ribeirão Preto, \\ Universidade de São Paulo, Ribeirão Preto, SP, Brasil \\ ${ }^{3}$ Departamento de Medicina Interna (Centro de Ciência da Imagem), Faculdade de Medicina de Ribeirão Preto, \\ Universidade de São Paulo, Ribeirão Preto, SP, Brasil
}

\begin{abstract}
The purpose of this study was to investigate the relationship between cyclin D1 expression and clinicopathological parameters in patients with prostate carcinoma. We assessed cyclin D1 expression by conventional immunohistochemistry in 85 patients who underwent radical prostatectomy for prostate carcinoma and 10 normal prostate tissue samples retrieved from autopsies. We measured nuclear immunostaining in the entire tumor area and based the results on the percentage of positive tumor cells. The preoperative prostate-specific antigen (PSA) level was $8.68 \pm 5.16 \mathrm{ng} / \mathrm{mL}$ (mean $\pm \mathrm{SD}$ ). Cyclin D1 staining was positive (cyclin D1 expression in $>5 \%$ of tumor cells) in 64 cases $(75.4 \%$ ) and negative (cyclin D1 expression in $\leq 5 \%$ of tumor cells) in 21 cases (including 15 cases with no immunostaining). Normal prostate tissues were negative for cyclin D1. Among patients with a high-grade Gleason score ( $\geq 7), 86 \%$ of patients demonstrated cyclin D1 immunostaining of $>5 \%(P<0.05)$. In the crude analysis of cyclin D1 expression, the high-grade Gleason score group showed a mean expression of $39.6 \%$, compared to $26.9 \%$ in the low-grade Gleason score group $(P<0.05)$. Perineural invasion tended to be associated with cyclin $\mathrm{D} 1$ expression $(P=0.07)$, whereas cyclin D1 expression was not associated with PSA levels or other parameters. Our results suggest that high cyclin D1 expression could be a potential marker for tumor aggressiveness.
\end{abstract}

Key words: Prostate cancer; Cyclin D1; Immunohistochemistry

\section{Introduction}

Prostate carcinoma is the most common malignant tumor in men older than 50 years of age and is characterized by a highly variable clinical course $(1,2)$. Accordingly, many potential prognostic markers have been extensively studied. Tumor markers enable cancer screening, differentiation between benign and malignant tumors, assessment of prognosis, therapeutic monitoring, and detection of tumor recurrence. Tumor markers have been closely evaluated to identify proteins that mediate and participate in tumor cell cycle progression.

Cyclin D1 is a short-lived nuclear protein that is degraded by the ATP ubiquitin-dependent proteolysis pathway and is involved in cell cycle transition from G1 (growth) to S phase (synthesis) in both normal and neoplastic cells $(3,4)$. Cyclin D1 overexpression prevents normal cell cycle regulation, causing uncontrolled cell proliferation, abnormal tissue growth, and transformation to a neoplastic phenotype, thereby acting as an oncogene (5). The relationship between cyclin D1 expression and prostate cancer remains unclear. Some studies have shown that cyclin D1 expression in prostate cancer is rare, whereas others report that prostate tumors with high cyclin D1 expression are associated with a more aggressive disease (6-8).

Because the role of cyclin D1 in prostate cancer is unclear, we studied the expression of cyclin D1 in prostate adenocarcinoma, to evaluate the relationship of this protein with epidemiological factors and clinicopathological features.

\section{Material and Methods}

\section{Clinical and pathological data}

From 2005 to 2010, we identified 450 patients with prostate carcinoma who underwent radical prostatectomy at Faculdade de Medicina de Ribeirão Preto, Universidade de São Paulo, Brazil. After exclusion criteria (samples with 
less than 2000 tumor cells) were applied, a total of 156 patients were eligible for analysis. From these, 43 cases with a high-grade Gleason score and 42 cases with a lowgrade Gleason score were randomly selected. All Gleason score information was acquired after radical prostatectomy. All necessary preoperative data regarding clinical, epidemiological, and histological features were acquired, and missing information was considered an exclusion criterion. Perineural invasion, angiolymphatic invasion, acinar atrophy, benign prostatic hyperplasia, and prostatic intraepithelial neoplasia were scored as positive or negative. Gleason scores were scored as low-grade (Gleason score $\leq 6$ ), and high-grade (Gleason score $\geq 7$ ). Pathological stage was scored as organ confined (without capsular tumor invasion and/or extracapsular) and extracapsular (with the presence of capsular tumor invasion and/or extracapsular). Tumor laterality was scored as unilateral (tumor affecting only one side of the prostate), and bilateral (tumor affecting both sides of the prostate). Preoperative prostate-specific antigen (PSA) was scored as less than $4 \mathrm{ng} / \mathrm{mL}$, from 4 to $10 \mathrm{ng} / \mathrm{mL}$, and greater than $10 \mathrm{ng} / \mathrm{mL}$. If less than 2000 tumor cells were detected during the count, the specimen was excluded from analysis. Tonsils were used as positive control tissue (according to the manufacturer's instructions), and normal prostate tissue with no sign of malignancy from autopsies of men $\leq 40$ years of age was also used. All cases were reviewed by two pathologists. The procedures were approved by the Ethics Committee of the Universidade de São Paulo.

\section{Immunohistochemistry and cyclin D1 analysis}

Immunohistochemistry was performed on 4- $\mu \mathrm{m}$ tissue sections using a polymer-based method and antigen retrieval. Cyclin D1 monoclonal antibodies (SP4 clone; Cell Marque, USA) were used. Briefly, sections were immersed in EDTA-Tris solution, $\mathrm{pH} \mathrm{9.0,} \mathrm{for} 30$ min at $95^{\circ} \mathrm{C}$. After cooling and washing, endogenous peroxidase activity was blocked and this was followed by blocking of nonspecific antibody sites. The sections were incubated with a solution of antibodies to cyclin D1 (1:200 dilution with $0.005 \%$ bovine serum albumin; Sigma, USA) for $2 \mathrm{~h}$. Diaminobenzidine was used as the final chromogen, and hematoxylin was used as a nuclear counterstain.

Nuclear immunostaining was assessed in the entire tumor area using conventional light microscopy (Nikon Eclipse 80i microscope, Nikon Digital Sight Camera DSU3, and DS-Fi1 and NIS Elements 3.22 software, Japan). Tumor samples with at least 2000 cells were counted at $200 \times$ magnification from randomly selected areas, in a blinded manner. All cells showing positive nuclear immunostaining were counted, regardless of intensity.

\section{Statistical analysis}

Receiver operating characteristic (ROC) curves were used to establish the optimum cut-off value for this study.
In order to perform statistical analysis, two groups of patients were divided according to Gleason scores: a high-grade group $(\geq 7)$ and a low-grade group $(\leq 6)$. Additionally, the relationships between cyclin D1 expression and perineural invasion, benign prostatic hyperplasia, PSA level, and other relevant clinicopathological parameters were evaluated. The chi-square test was used to compare the frequency of categorical variables between groups, the Mann-Whitney test was used to compare continuous variables with nonnormal distributions, and Spearman correlation was used to evaluate the relationship between PSA levels and cyclin D1 expression.

\section{Results}

The patient age was $67.7 \pm 7.0$ years (mean \pm SD) and the preoperative PSA level was $8.68 \pm 5.16 \mathrm{ng} / \mathrm{mL}$ (mean \pm SD). The patient population comprised $79 \%$ Caucasian men, and $60 \%$ were patients with a tumor node metastasis (TNM) stage of pT2c. Four patients showed metastatic disease and one died due to prostate cancer.

Table 1 summarizes the relationship between immunohistochemistry data and clinicopathological parameters. Perineural invasion tended to be associated with cyclin D1 expression $(P=0.07)$, and 39 cases $(60 \%)$ with perineural invasion were positive for cyclin D1 expression. Other histological parameters including acinar atrophy, benign prostatic hyperplasia, lymphovascular invasion, and prostatic intraepithelial neoplasia were not associated with cyclin D1 expression (Table 1).

Figure 1 illustrates the immunohistochemistry staining pattern of cyclin D1 expression in normal prostate and prostate carcinoma tissue. Positive staining for cyclin D1 was detected in 64 cases $(75.4 \%)$. Among the 21 cyclin D1-negative cases, 15 showed no cyclin D1 immunostaining. The mean percentage of immunostaining was $44 \%$ in the cyclin D1-positive group and $1.4 \%$ in the cyclin D1negative group. All normal prostate tissues (10 cases) assessed showed no immunostaining for cyclin D1 (Figure 1A). Cyclin D1-positive prostate carcinomas displayed a heterogeneous staining pattern for cyclin D1, with high and low immunostaining within the same focal field (Figure 1C). Figure $1 \mathrm{~B}$ is a representative image of a low-grade tumor negative for cyclin D1 staining.

Patients were divided into two groups according to the cut-off value determined by ROC curve analysis: cyclin D1-positive patients (cyclin D1 expression in $>5 \%$ of tumor cells) and cyclin D1-negative patients (cyclin D1 expression in $\leq 5 \%$ of tumor cells). For statistical purposes, the cut-off value of $5 \%$ for cyclin D1 expression had a high sensitivity, but low specificity (Table 2 and Figure 2). Of the cases with a high-grade Gleason score $(\geq 7), 86 \%$ exhibited cyclin D1 immunostaining of $>5 \%(P<0.05)$. In a crude analysis, the high-grade Gleason score group had a mean percentage of cyclin D1 expression of $39.6 \%$, 
Table 1. Relationship between clinicopathological parameters and cyclin D1 expression in prostate carcinoma.

\begin{tabular}{|c|c|c|c|}
\hline Parameter & Cyclin D1 $\leq 5 \%$ & Cyclin D1 >5\% & Total \\
\hline Total & $21(24.7 \%)$ & $64(75.3 \%)$ & 85 \\
\hline \multicolumn{4}{|l|}{ Gleason score } \\
\hline Low-grade & $15(35.71 \%)^{*}$ & $27(64.29 \%)$ & 42 \\
\hline High-grade & $6(13.95 \%)$ & $37(86.05 \%)$ & 43 \\
\hline \multicolumn{4}{|l|}{ Perineural invasion } \\
\hline Negative & $13(61.9 \%)$ & $25(39.06 \%)$ & 38 \\
\hline Positive & $8(38.1 \%)$ & $39(60.94 \%)$ & 47 \\
\hline \multicolumn{4}{|c|}{ Angiolymphatic invasion } \\
\hline Negative & $21(26.25 \%)$ & $59(73.75 \%)$ & 80 \\
\hline Positive & $0(0.0 \%)$ & $5(100.0 \%)$ & 5 \\
\hline \multicolumn{4}{|l|}{ Pathological stage } \\
\hline Organ confined & $18(27.7 \%)$ & $47(72.3 \%)$ & 65 \\
\hline Extracapsular & $3(15.0 \%)$ & $17(85.0 \%)$ & 20 \\
\hline \multicolumn{4}{|l|}{ Tumor laterality } \\
\hline Unilateral & $4(28.57 \%)$ & $10(71.43 \%)$ & 14 \\
\hline Bilateral & $17(23.94 \%)$ & $54(76.06 \%)$ & 71 \\
\hline \multicolumn{4}{|c|}{ Prostatic intraepithelial neoplasia } \\
\hline Negative & $13(29.54 \%)$ & $31(70.46 \%)$ & 44 \\
\hline Positive & $8(19.5 \%)$ & $33(80.5 \%)$ & 41 \\
\hline \multicolumn{4}{|c|}{ Preoperative PSA (ng/mL) } \\
\hline$<4$ & $1(11.1 \%)$ & $8(88.9 \%)$ & 9 \\
\hline $4-10$ & $15(28.85 \%)$ & $37(71.15 \%)$ & 52 \\
\hline$>10$ & $5(20.83 \%)$ & $19(79.17 \%)$ & 24 \\
\hline \multicolumn{4}{|c|}{ Benign prostatic hyperplasia } \\
\hline Negative & $5(23.8 \%)$ & $16(76.2 \%)$ & 21 \\
\hline Positive & $16(25 \%)$ & $48(75 \%)$ & 64 \\
\hline \multicolumn{4}{|l|}{ Acinar atrophy } \\
\hline Negative & $11(21.15 \%)$ & $41(78.85 \%)$ & 52 \\
\hline Positive & $10(30.3 \%)$ & $23(69.7 \%)$ & 33 \\
\hline
\end{tabular}

Data are reported as number with percent in parentheses. PSA: prostate-specific antigen. ${ }^{*} \mathrm{P}<0.02$, low-grade compared to high-grade Gleason score (chi-square test).

compared to $26.9 \%$ in the low-grade Gleason score group $(\mathrm{P}<0.05$; Table 3 and Figure 3).

As shown in Figure 4, cyclin D1 expression was not associated with PSA levels according to Spearman correlation analysis $(P=0.90)$. However, approximately $80 \%$ of cases with preoperative PSA levels greater than $10 \mathrm{ng} / \mathrm{mL}$ displayed $>5 \%$ cyclin $\mathrm{D} 1$ expression $(P=0.46)$.

\section{Discussion}

In this study, we assessed the relationship between cyclin D1 expression and clinicopathological features in 85 patients with a diagnosis of prostate carcinoma who underwent radical prostatectomy. The mean age and mean preoperative PSA levels were similar to those reported in previous studies $(7,9,10)$. The predominant pathological TNM stage was pT2c $(60 \%)$, but this was different from previous studies by Freedland et al. (11) (pT2a, 34\%) and Makarov et al. (12) (pT1c, 77\%). Our findings are in agreement with those of Nardi et al. (13) who reported that patients treated in a public health care institution, as was the case in our study, represent a cohort with specific characteristics such as higher PSA levels, slightly older age, and more advanced disease than patients treated in a private health care institution (13).

PSA is considered a nonspecific biomarker for prostate cancer, associated with a high false-positive index (approximately $50 \%$ ) and a $15 \%$ false-negative index (14). Our study provides further evidence for the lack of a correlation between PSA levels and cyclin D1 expression, thereby supporting the theory that PSA levels are not associated with cell cycle disorders in prostate carcinoma. However, studies such as those by Drobnjak et al. (6), Kallakury et al. (15), Comstock et al. (16), and Ilia Anis et al. (17) have found statistically significant positive and negative correlations of cyclin D1 expression and PSA levels. Nakamura et al. (10) reported that cyclin D1 expression might be regulated by sex hormones, indicating 

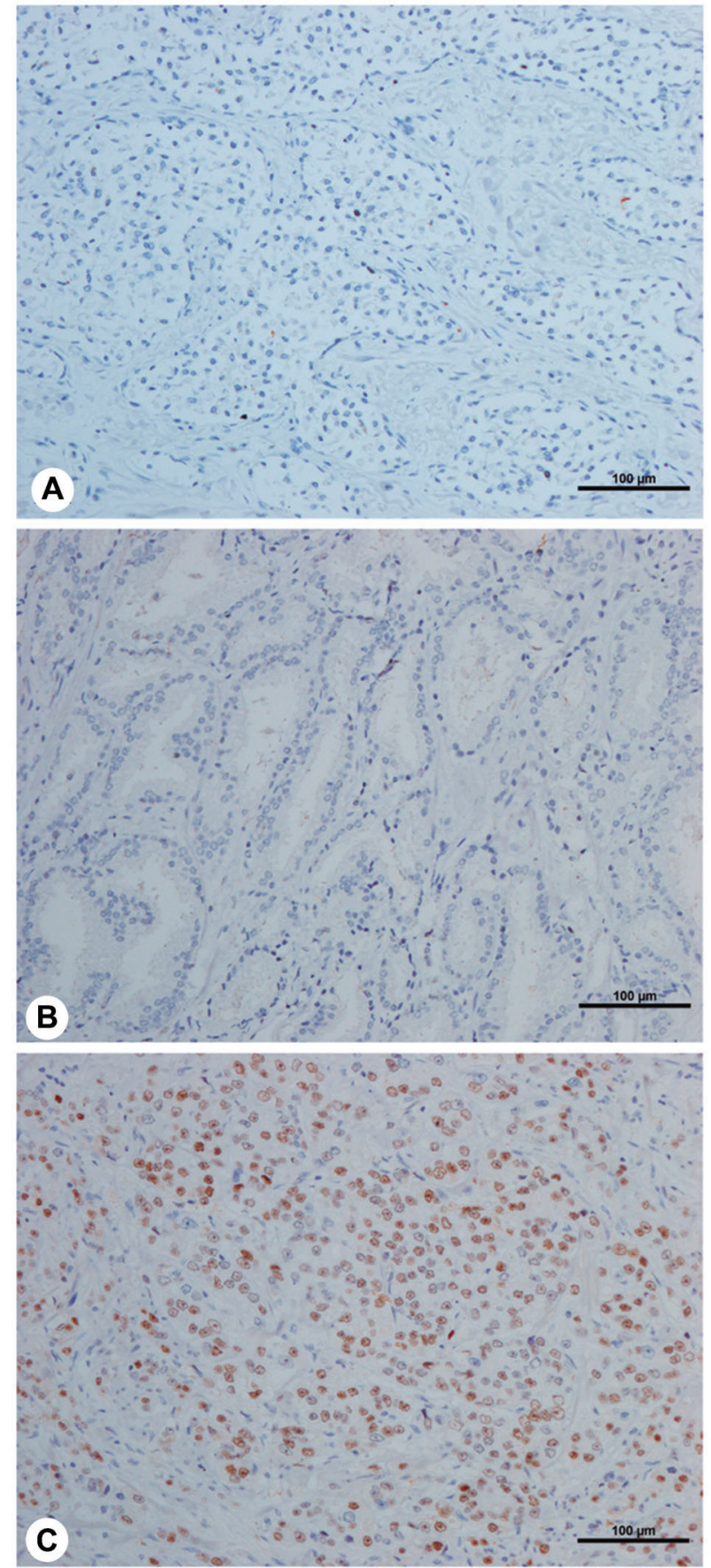

Figure 1. Cyclin D1 immunohistochemistry. $A$, Negative cyclin D1 immunostaining in normal prostate tissue. $B$, Immunostaining in cyclin D1-negative prostate carcinoma. $C$, Heterogeneous staining in cyclin D1-positive prostate carcinoma.

a possible relationship between PSA levels and cyclin D1, since PSA production is dependent on hormonal stimulation (1). Nonetheless, further studies are needed to determine the true relationship between PSA levels and
Table 2. Receiver operating characteristic curves for Gleason score.

\begin{tabular}{lccc}
\hline Cut-off & Sensitivity & Specificity & 1-Specificity \\
\hline 0 & 1 & 0 & 1 \\
5 & 0.907 & 0.262 & 0.738 \\
10 & 0.86 & 0.357 & 0.643 \\
15 & 0.791 & 0.429 & 0.571 \\
20 & 0.721 & 0.476 & 0.524 \\
25 & 0.698 & 0.5 & 0.5 \\
30 & 0.628 & 0.5 & 0.5 \\
35 & 0.558 & 0.571 & 0.429 \\
40 & 0.465 & 0.738 & 0.262 \\
45 & 0.395 & 0.762 & 0.238 \\
50 & 0.372 & 0.762 & 0.238 \\
55 & 0.256 & 0.786 & 0.214 \\
60 & 0.233 & 0.786 & 0.214 \\
65 & 0.233 & 0.833 & 0.167 \\
70 & 0.233 & 0.929 & 0.071 \\
75 & 0.186 & 0.952 & 0.048 \\
80 & 0.14 & 0.976 & 0.024 \\
85 & 0.116 & 1 & 0 \\
90 & 0.07 & 1 & 0 \\
95 & 0.047 & 1 & 0 \\
100 & 0.023 & 1 & 0 \\
AUC (95\%Cl) & & $63.62 \%(51.82-75.42 \%)$ \\
\hline
\end{tabular}

AUC: Area under the curve.

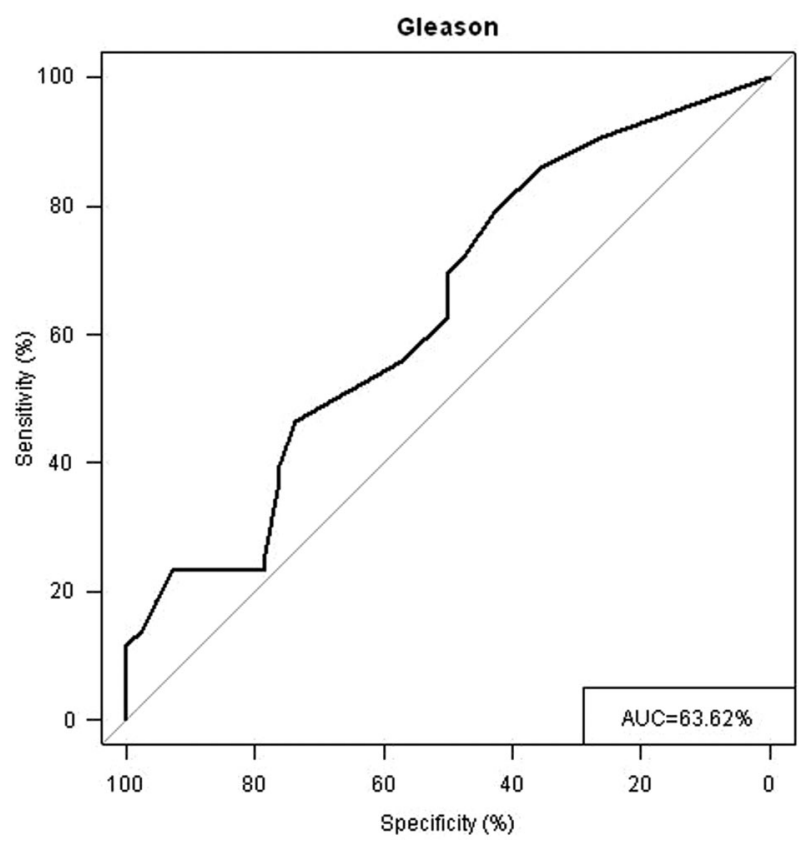

Figure 2. Receiver operating characteristic curve analysis for Gleason scores. AUC: area under the curve. 
Table 3. Cyclin D1 expression and Gleason score.

\begin{tabular}{lccccr}
\hline Gleason score & $\mathrm{N}$ & \multicolumn{3}{c}{ Cyclin D1 expression } \\
\cline { 3 - 5 } & & Mean \pm SD & Minimum & Median & Maximum \\
\hline Low-grade & 42 & $26.9 \pm 25.78^{*}$ & 0 & 25 & 80 \\
High-grade & 43 & $39.6 \pm 28.73$ & 0 & 35 & 100 \\
\hline
\end{tabular}

${ }^{*} \mathrm{P}<0.03$, low-grade compared to high-grade Gleason score (Mann-Whitney test).

cyclin D1 expression in order to confirm these hypotheses.

The heterogeneous pattern of cyclin D1 immunostaining observed in this study is in agreement with the results of Han et al. (18), who demonstrated $5-10 \%$ focal immunostaining, supporting the hypothesis that many different mechanisms are involved in prostate carcinogenesis (19). We also found that cyclin D1 expression tended to be associated with perineural invasion. Perineural invasion is considered an aggressive histological marker in prostate cancer, and a positive correlation between this histological feature and cyclin D1 expression has been extensively reported in studies of cyclin D1 and prostate cancer $(9,10)$. Donnellan and Chetty (5), in a review article, showed that many researchers found that intratumoral cyclin D1 levels were correlated with the outcome of prognosis for different malignancies. In most cases, higher expression of cyclin D1 was correlated with poor prognosis. In breast cancer, however, the expression of cyclin D1 was related to sex steroid receptor positivity, favorable histological signs, and good prognosis $(5,20)$.

Previous studies $(6,7)$ have shown no relationship

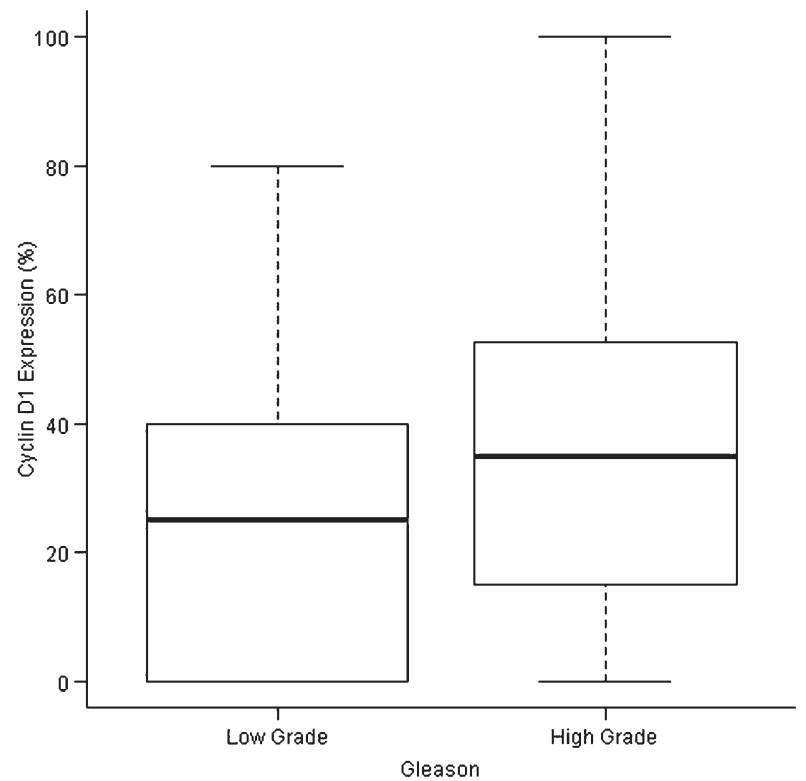

Figure 3. Distribution of Gleason scores according to cyclin D1 expression. between cyclin D1 expression and the Gleason score, but, in this study, we found a positive statistically significant association between the Gleason score and cyclin D1 expression, with high-grade tumors displaying high cyclin D1 expression compared with low-grade tumors, in agreement with a study conducted by Emin Özbek et al. (21). However, unlike our study, other studies may not have determined the optimal cut-off value using ROC curve analysis, such as the Emin Özbek study, that showed positive correlation between Gleason score and cyclin D1. In addition, cyclin D1-positive prostate cancer cells have been reported to show greater motility, increased invasion capability, and a hormone-independent phenotype in cell cultures, supporting the hypothesis that cyclin D1 plays an important role in aggressive prostate carcinogenesis $(22,23)$. These data and the relationship between perineural invasion and cyclin D1 expression reinforce the important role of this cell cycle protein in the prediction of more aggressive disease.

Taken together, our results and previous data show that cyclin D1 might indicate a more aggressive phenotype

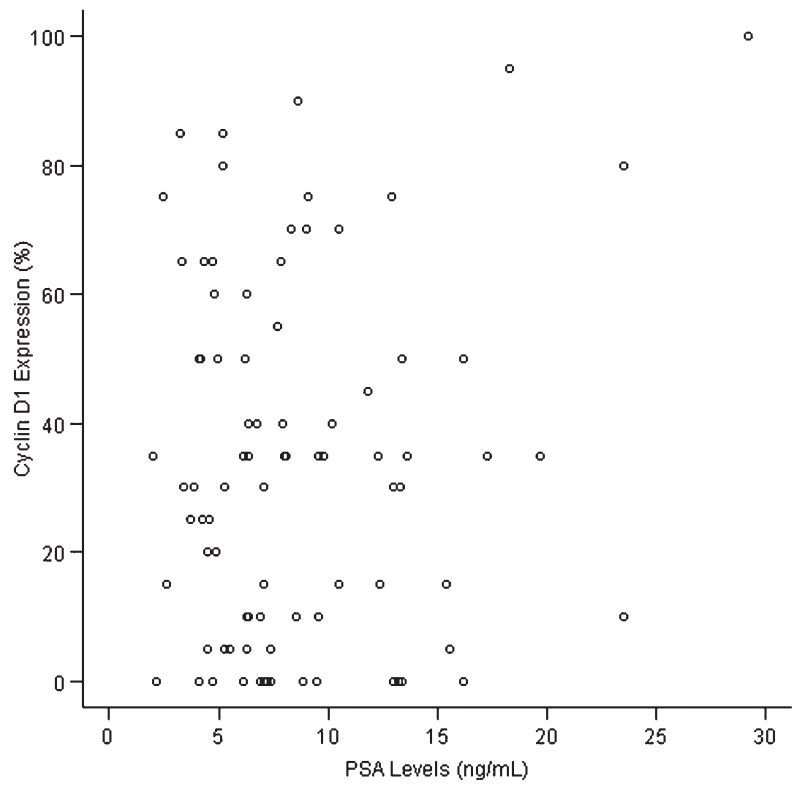

Figure 4. Relationship between cyclin D1 expression and preoperative prostate-specific antigen (PSA) levels. 
of prostate cancer and could perhaps be used as a new biomarker, particularly because of the nonspecific nature of PSA and other elements as cancer markers $(9,22-26)$. The Gleason score is the most widely used predictive preoperative histological parameter of tumor spread in urological practice and is considered capable of predicting tumor behavior, with the ability to differentiate between aggressive and benign disease $(24,25)$. However, previous studies (27-29) and our ongoing study indicate that the Gleason score is likely underestimated by preoperative biopsy of the prostate in approximately $40 \%$ of cases, resulting in false-negative results. This may hinder the accurate diagnosis of benign vs aggressive prostate cancer (27-29).

\section{References}

1. Dunn MW, Kazer MW. Prostate cancer overview. Semin Oncol Nurs 2011; 27: 241-250, doi: 10.1016/j.soncn.2011.07. 002.

2. INCA. Estimativa 2014. In: Ministério da Saúde (Editor), Incidência de câncer no Brasil. Rio de Janeiro: INCA; 2014. http://www.inca.gov.br/estimativa/2014/estimativa-24012014. pdf.

3. Baldin V, Lukas J, Marcote MJ, Pagano M, Draetta G. Cyclin D1 is a nuclear protein required for cell cycle progression in G1. Genes Dev 1993; 7: 812-821, doi: 10.1101/gad.7.5.812.

4. Diehl JA. Cycling to cancer with cyclin D1. Cancer Biol Ther 2002; 1: 226-231.

5. Donnellan R, Chetty R. Cyclin D1 and human neoplasia. Mol Pathol 1998; 51: 1-7, doi: 10.1136/mp.51.1.1.

6. Drobnjak M, Osman I, Scher HI, Fazzari M, Cordon-Cardo C. Overexpression of cyclin D1 is associated with metastatic prostate cancer to bone. Clin Cancer Res 2000; 6: 18911895.

7. Fleischmann A, Rocha C, Saxer-Sekulic N, Zlobec I, Sauter G, Thalmann GN. High-level cytoplasmic cyclin D1 expression in lymph node metastases from prostate cancer independently predicts early biochemical failure and death in surgically treated patients. Histopathology 2011; 58: 781789, doi: 10.1111/j.1365-2559.2011.03800.x.

8. Kolar Z, Murray PG, Scott K, Harrison A, Vojtesek B, Dusek $\mathrm{J}$. Relation of $\mathrm{Bcl}-2$ expression to androgen receptor, p21WAF1/CIP1, and cyclin D1 status in prostate cancer. Mol Pathol 2000; 53: 15-18, doi: 10.1136/mp.53.1.15.

9. Aaltomaa S, Karja V, Lipponen $\mathrm{P}$, Isotalo $\mathrm{T}$, Kankkunen JP, Talja M, et al. Expression of Ki-67, cyclin D1 and apoptosis markers correlated with survival in prostate cancer patients treated by radical prostatectomy. Anticancer Res 2006; 26: 4873-4878.

10. Nakamura $\mathrm{Y}$, Felizola SJ, Kurotaki $\mathrm{Y}$, Fujishima $\mathrm{F}$, McNamara KM, Suzuki T, et al. Cyclin D1 (CCND1) expression is involved in estrogen receptor beta (ERbeta) in human prostate cancer. Prostate 2013; 73: 590-595, doi: 10.1002/pros.22599.

11. Freedland SJ, Humphreys EB, Mangold LA, Eisenberger M, Dorey FJ, Walsh PC, et al. Risk of prostate cancer-specific mortality following biochemical recurrence after radical
In summary, our data show variable expression of cyclin D1 among prostate carcinoma cells and absence in normal prostate tissue. We found that high expression of cyclin D1 $(>5 \%)$ was associated with a high-grade Gleason score $(\geq 7)$ and the presence of perineural invasion. Because of the likelihood of underestimating the Gleason score by prostate biopsy and the positive relationship demonstrated in our study between cyclin D1 expression and the Gleason score, we suggest that the use of cyclin D1 immunohistochemistry in prostate cancer may decrease the likelihood of underestimating tumor aggressiveness. However, studies using other approaches are needed to elucidate the efficacy of cyclin D1 as a tumor marker or therapeutic target in prostate cancer.

prostatectomy. JAMA 2005; 294: 433-439, doi: 10.1001/ jama.294.4.433.

12. Makarov DV, Trock BJ, Humphreys EB, Mangold LA, Walsh $\mathrm{PC}$, Epstein $\mathrm{Jl}$, et al. Updated nomogram to predict pathologic stage of prostate cancer given prostate-specific antigen level, clinical stage, and biopsy Gleason score (Partin tables) based on cases from 2000 to 2005. Urology 2007; 69: 1095-1101, doi: 10.1016/j.urology.2007.03.042.

13. Nardi AC, Reis RB, Zequi Sde C, Nardozza A Jr. Comparison of the epidemiologic features and patterns of initial care for prostate cancer between public and private institutions: a survey by the Brazilian Society of Urology. Int Braz J Urol 2012; 38: 155-164, doi: 10.1590/S1677-55382 012000200003.

14. Thompson IM, Pauler DK, Goodman PJ, Tangen CM, Lucia $\mathrm{MS}$, Parnes $\mathrm{HL}$, et al. Prevalence of prostate cancer among men with a prostate-specific antigen level $<$ or $=4.0 \mathrm{ng}$ per milliliter. N Engl J Med 2004; 350: 2239-2246, doi: 10.1056/ NEJMoa031918.

15. Kallakury BV, Sheehan CE, Ambros RA, Fisher HA, Kaufman RP Jr, Ross JS. The prognostic significance of p34cdc2 and cyclin D1 protein expression in prostate adenocarcinoma. Cancer 1997; 80: 753-763, doi: 10.1002/ (SICI)1097-0142(19970815)80:4<753::AID-CNCR15>3.0. CO;2-S.

16. Comstock CE, Revelo MP, Buncher CR, Knudsen KE. Impact of differential cyclin D1 expression and localisation in prostate cancer. $\mathrm{Br} J$ Cancer 2007; 96: 970-979, doi: 10.1038/sj.bjc.6603615.

17. Ilia Anis $\mathrm{HNH}$, Darweesh MF, Abd El Rahman M. Immunohistochemical expression of Cyclin D1 in Egyptian patients with prostatic carcinoma. World J Med Sci 2013; 8: 306-313.

18. Han EK, Lim JT, Arber N, Rubin MA, Xing WQ, Weinstein IB. Cyclin D1 expression in human prostate carcinoma cell lines and primary tumors. Prostate 1998; 35: 95-101, doi: 10.1002/(SICI)1097-0045(19980501)35:2<95::AIDPROS2>3.0.CO;2-F.

19. Aquino G, Franco R, Ronconi F, Anniciello A, Russo L, De Chiara A, et al. Peripheral T-cell Lymphoma with Cyclin D1 overexpression: a case report. Diagn Pathol 2012; 7: 79, doi: 10.1186/1746-1596-7-79. 
20. Gillett C, Smith P, Gregory W, Richards M, Millis R, Peters $\mathrm{G}$, et al. Cyclin D1 and prognosis in human breast cancer. Int J Cancer 1996; 69: 92-99, doi: 10.1002/(SICl)10970215(19960422)69:2<92::AID-IJC4>3.0.CO;2-Q.

21. Emin Özbek BM, Mustafa Özbek, Süleyman Büyükberber MD. Cyclin-D1 protooncogen expression in prostate cancer. Turkish J Cancer 2000; 30.

22. Ding GX, Liu J, Feng CC, Jiang HW, Xu JF, Ding Q. Slug regulates Cyclin D1 expressionin by ubiquitin-proteasome pathway in prostate cancer cells. Panminerva Med 2012; 54: 219-223.

23. He Y, Franco OE, Jiang M, Williams K, Love HD, Coleman IM, et al. Tissue-specific consequences of cyclin D1 overexpression in prostate cancer progression. Cancer Res 2007; 67: 8188-8197, doi: 10.1158/0008-5472.CAN-07-0418.

24. Koontz BF, Tsivian M, Mouraviev V, Sun L, Vujaskovic Z, Moul J, et al. Impact of primary Gleason grade on risk stratification for Gleason score 7 prostate cancers. Int $J$ Radiat Oncol Biol Phys 2012; 82: 200-203, doi: 10.1016/ j.jijobp.2010.11.023.

25. Lughezzani G, Gallina A, Larcher A, Briganti A, Capitanio U, Suardi $N$, et al. Radical prostatectomy represents an effective treatment in patients with specimen-confined high pathological Gleason score prostate cancer. BJU Int 2013; 111: 723-730, doi: 10.1111/j.1464-410X.2012.11114.x.

26. Becker C, Noldus J, Diamandis E, Lilja H. The role of molecular forms of prostate-specific antigen (PSA or hK3) and of human glandular kallikrein 2 (hK2) in the diagnosis and monitoring of prostate cancer and in extra-prostatic disease. Crit Rev Clin Lab Sci 2001; 38: 357-399, doi: 10.1080/20014091084236.

27. Brawer MK, Crawford ED, Fowler J, Lucia MS, Schroeder FH. Prostate cancer: epidemiology and screening. Rev Urol 2000; 2 (Suppl 4): S5-S9.

28. Cookson MS, Fleshner NE, Soloway SM, Fair WR. Correlation between Gleason score of needle biopsy and radical prostatectomy specimen: accuracy and clinical implications. J Urol 1997; 157: 559-562, doi: 10.1016/ S0022-5347(01)65201-7.

29. Kulkarni JN, Valsangkar RS, Jadhav YR, Singh DP. Impact of Gleason pattern up gradation after radical prostatectomy for carcinoma prostate patients with low biopsy score $(\leq 6)$. J Cancer Res Ther 2011; 7: 459-462, doi: 10.4103/09731482.92021. 